\title{
Preparation of Graphene Nanosheets from Graphite Flakes via Shear Assisted Exfoliation
}

\author{
Ali J. Saloum, Basma H. Al-Tamimi, Saad B.H. Farid $\square$ \\ Materials Engineering Dept., University of Technology-Iraq, Alsina'a street,10066 Baghdad, Iraq. \\ *Corresponding author Email: mae.19.13@grad.outechnology.edu.iq
}

\section{H I G H L I G H T S}

- Graphene nanosheets were prepared via a series of mechanical exfoliation methods.

- Raman spectra confirm that obtained graphene has the fewest number of layers.

- According to the result of XRD, the obtained graphene layer has a thickness of $0.34 \mathrm{~nm}$.

- The route used is a way of producing lowcost graphene at a high production rate.

\section{A R T I C L E IN F O}

Handling editor: Jawad K. Oleiwi

\section{Keywords:}

Graphene

Graphite

Ball milling

Shear force

Ultra-sonication

Raman spectra

\author{
A B S T R A C T
}

\begin{abstract}
Graphene is one of the most important forms of carbon. Due to its exceptional physical, chemical, and mechanical properties, it's used in different fields such as electronic, energy storage, and medical applications. Therefore, the production of graphene in large quantities, at a low cost, and high quality, has become critical. Using graphite powder, a few layers of graphene sheets were prepared via a series of mechanical exfoliation methods. the dry ball milling process by the planetary mill was used. Thereafter, the milling was followed by shear force and then supported by ultra-sonication to reach the lowest level of the graphene layers. Morphological properties were examined using a scanning electron microscope (SEM) and a transmission electron microscope (TEM). Also, structural characterizations were investigated using Raman spectra and X-ray diffraction measurements (XRD) and (FTIR). Findings presented in this research highlighted that the synthesis method followed was found to have several advantages, including low cost and the ease of producing a few layers of graphene nanosheets. Subsequently, the promising efficiency of the used methodology is important.
\end{abstract}

\section{Introduction}

Carbon is one of the most common elements with structural varieties on the periodic table [1]. Because of its unique electronic structure, each carbon atom may be linked to single, double, or triple covalent bonds with another carbon atom or another element. The electronic configuration of the carbon atom affects its properties as well as its ability to exist in a variety of forms known as allotropes [2]. The outcome of different hybridization states of carbon ( $\mathrm{sp}, \mathrm{sp}^{2}$, and $\left.\mathrm{sp}^{3}\right)$. Many allotropes were produced, such as diamond $\left(\mathrm{sp}^{3}\right)$, graphite $\left(\mathrm{sp}^{2}\right)$, and graphene $\left(\mathrm{sp}^{2}\right)$. As a result, carbon-based materials, particularly graphene, have emerged as having scientific and technological significance, attracting massive funding for project initiatives while also being researched-intensive [3]. Since Prof. A. Geim and Prof. Novoselov revolutionary discovery in 2004 [4]. Graphene has emerged to become a thrilling 2D material with distinct attributes that have attracted great interest in the fields of chemistry, physic, as well as their related interdisciplinary [5]. Graphene, as the thinnest material existing, the thickness of one carbon atom arranged in a hexagonal lattice resembling a honeycomb, with bonded carbon atoms distance $1.42 \mathrm{~A}^{\circ}$, has unique properties like mechanical flexibility, electrical, and thermal conductivity [6]. Ultimately, graphene became a "rising star" on the horizon of materials science [7]. The $\mathrm{sp}^{2}$ covalent bonds $(\sigma \mathrm{C}-\mathrm{C})$ give graphene its mechanical strength, and the orbitals $(\pi)$ make graphene conduct. So, it has become clear that the properties of graphene strongly depend on its unique structure and configuration [8]. Further, it is the cornerstone in the formation of carbon allotropes. graphene can compact and form threedimensional graphite, or it can be rolled to form a one-dimensional carbon nanotube or wound around an axis and form a zerodimensional fluorine ball [9].

There are several techniques used to produce graphene. Some of them belong to top-down methods such as the exfoliation method and chemical method, usually this method tends to produce graphene in high quantity but with some structural defects that limit its application [10]. Other techniques belong to bottom-up methods such as the chemical vapor deposition (CVD) 
method which is characterized by its ability to produce graphene with high quality and controlled properties but it is suffering from the low-scalability and high-cost requirements [11].

W. Zhao et al. argued on graphene synthesis via exfoliated graphite by ball milling with shear forces inside the planetary mill chamber [12]. E. Varrla et al. prepared graphene from graphite powder by shear force. The shear force leads to the fragmentation of the graphite layers by sufficient energy to overcome (Vander Waals forces) between the graphite layers to obtain a few layers of graphene [13]. Z. Ismail et al. Using a kitchen mixer and commercial black tea, graphene was prepared by direct exfoliation of graphite. Meanwhile, using a kitchen mixer to exfoliate graphene does not create any basal defects. For the exfoliated graphene, in addition, it suggests that aliphatic compounds in tea may be responsible for the exfoliation and stabilization of graphene in water [14]. Y. Htwe et al. graphene was produced with the highest stability, best electrical conductivity, and fewer defects. Ultra-sonication at room temperature was used to assist in the electrochemical exfoliation of graphene. by using different types of electrolytes [15]. G. Huang et al. high-quality graphene was successfully produced by mechanically exfoliating graphite flakes in a ball mill, and layered multi-layered graphene was produced using a $1200 \mathrm{r} / \mathrm{min}$ vibration frequency and stainless-steel grit [16].

This work aims to synthesis two-dimensional graphene following mechanical exfoliation and using hybrid method (mechanical exfoliation by balls, shear blades, and ultra-sonication) to achieve mass production of graphene nanosheets.

\section{Materials and Methods}

\subsection{Materials}

An elemental graphite powder utilized in preparing graphene nanosheets was obtained from (central drug house/New Delhi/India) and the ethanol was obtained from (pronto chemical Co. turkey, 96\% pure).

\subsection{Methods}

The starting-up powder graphite was weighed $(10 \mathrm{~g})$. The graphite was milled via a dry ball milling process that was carried out using a planetary ball mill. The grinding media were hardened steel balls. The ball milling was run mild milling speed of $(600 \mathrm{rpm})$ [17] for $6 \mathrm{~h}$. In this process, the moving balls apply their kinetic energy to the material, break chemical bonding between the sheets, and produce new surfaces [18]. There are two possible mechanisms responsible for the exfoliation and fragmentation effects. The first is shear force, which is thought to be a brilliant mechanical method of exfoliation that allows graphene sheets to delaminate, While the second one is the collisions or vertical impacts applied by the balls during rolling actions. Planetary ball milling has the advantage of having a mass production rate [19].

To ensure the generation of shear forces which play an important role in the exfoliation of graphite sheets. The rotatingblade mixer is the second stage adopted in this study. The produced graphite powder from the ball mill method was dispersed in $400 \mathrm{ml}$ ethanol solution dispersions in a kitchen blender with a three-blade impeller ( $3000 \mathrm{rpm}), 1800 \mathrm{~W}$ for $2 \mathrm{~h}$. The shear exfoliation depends on the blades of the mixer, which motion blades generate shear, turbulence, and collisions with the assistance of fluid dynamics and its cooperative effect in the events of the exfoliation through graphite self-lubricating ability in the lateral direction [20].

Finally, the ultra-sonication technique was used to improve the reduction of graphene sheets generated from the previous two stages. The mixture obtained from the second stage was used by the Ultra-sonication device, (Cup Tip, 20kHz, model 300 $\mathrm{VT}$ ), for $2 \mathrm{~h}$. In the solution, it will cause alternating compression and permeate cycles. During the pressure cycle, small bubbles would form and these would grow and reach a size that could not absorb more energy and they would collapse. As a result of this phenomenon, graphene layers will exfoliate [21]. After completing the process, the solution is dried in the furnace at $90^{\circ} \mathrm{C}$ for $6 \mathrm{~h}$.

Figure 1 shows the stages of the whole process, starting from the stage of milling graphite powder in the planetary mill, where the principle of balls grinds the layers from the top and bottom of graphite followed by the second stage, using a rotating-blade mixer to obtain sufficient shear force to mill the layers of graphene to reach the lowest layers. The last stage represents ultra-sonication to obtain graphene. The above paragraphs are characterized by low energy and also a reduction in the use of chemicals that affect the structure of graphene.

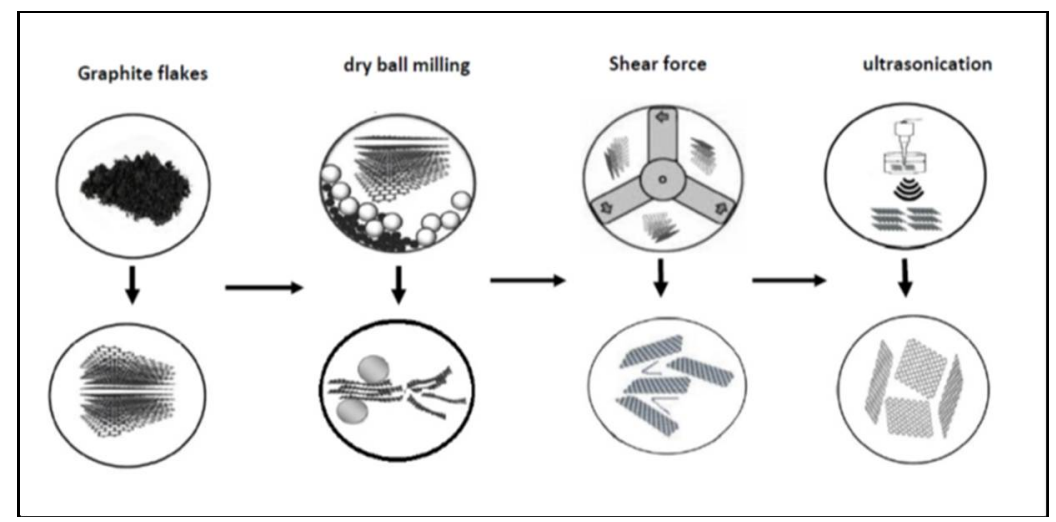

Figure 1: Represents the series of steps involved in preparing graphene 


\section{Results and Discussion}

Raman spectrometer is one of the most important non-destructive testing techniques for the sample in addition to being easy and fast and providing direct insight into the interaction between the electron and the photon. The spectrometer is characterized by determining the quality and structure of carbon nanomaterials as well as defects and disorders. The

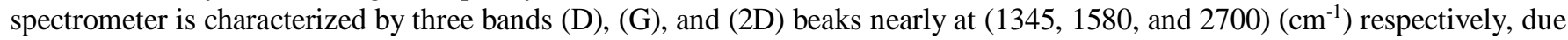
to the change of electron bands [22].

The band or beak (D) indicates defects and disorders in the structure. As it tends to be weak in graphite structure and high in quality in graphene, it can be observed in Figures 2 and 3. It was also noticed the decreasing of the D intensity for the graphite band around $1346\left(\mathrm{~cm}^{-1}\right)$ and the graphene prepared for $1350\left(\mathrm{~cm}^{-1}\right)$. By comparing the two planners for graphene and graphite it was observed that the D intensity of graphene is higher than the intensity for graphite, due to the presence of some defects that resulted from dry milling and shear force. So, the intensity of the defects is directly proportional to the presence of defects.

The band $(\mathrm{G})$ is the main range in the Raman spectrometer, through which it expresses the thickness of the layer of graphene compared to the graphite, as it is a vibration band within the plane related to the sp2 hybrid carbon atoms that consist of the graphene sheets, and their examination gives an accurate indication of the thickness of the layer. The $G$ range for graphite is about $1572\left(\mathrm{~cm}^{-1}\right)$ higher than $1576\left(\mathrm{~cm}^{-1}\right)$ for graphene. This indicates the thickness of the graphite layers compared to graphene. The results showed the efficiency of the graphene preparation that used in this work.

The band (2D) is twice the frequency of the D-band in the Raman spectrometer, as it is $2705\left(\mathrm{~cm}^{-1}\right)$ in graphite and 2703 $\left(\mathrm{cm}^{-1}\right)$ in graphene. The measurement refers to the thickness of the graphene layers, which indicates according to the mentioned ranges and shown in the diagram that graphene is the least number of layers of graphite. Therefore, it is deduced from the ability of the technique to produce graphene.

It is well documented that the use of different chemicals in the preparation of two-dimension graphene is avoided because they could affect the structure and physico-chemical properties of graphene, especially electrical and conductivity. Further, it is also reported to avoid the use of high speed in dry milling as it also affects the structure and fracture of planes. On the other hand, to examine the morphological structure of graphite, and graphene prepared, graphene was examined by scanning electron microscope (SEM). Figure 4 refers to the (SEM) micrographs of the graphene obtained from the graphite flakes. The micrograph (a) graphite flakes are different from the thin, paper-like shape of the produced graphene in (b). Figure 5 displays the typical result of the transmission electron microscopy (TEM) image of the prepared graphene, from this figure the wide, flat, thin, and paper-like shape of the prepared two-dimensional material via the simple and facial method. Also, the presence of the graphene structure can be observed as a single-layer sheet and in other areas as a double-layer sheet. So, from this analysis result, the used route can be regarded as a route to produce graphene with a single and few layers.

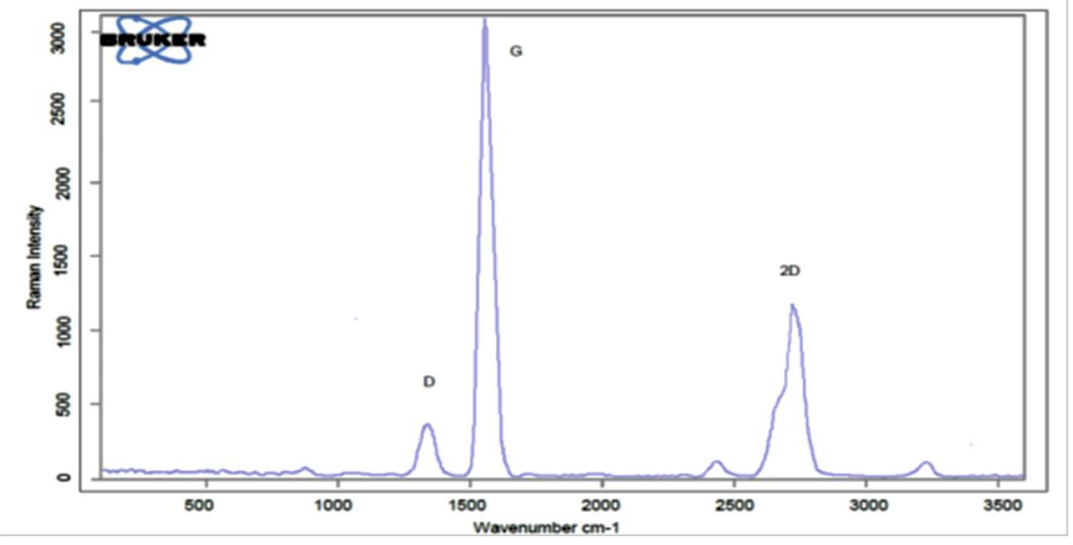

Figure 2: Raman spectra of the used graphite

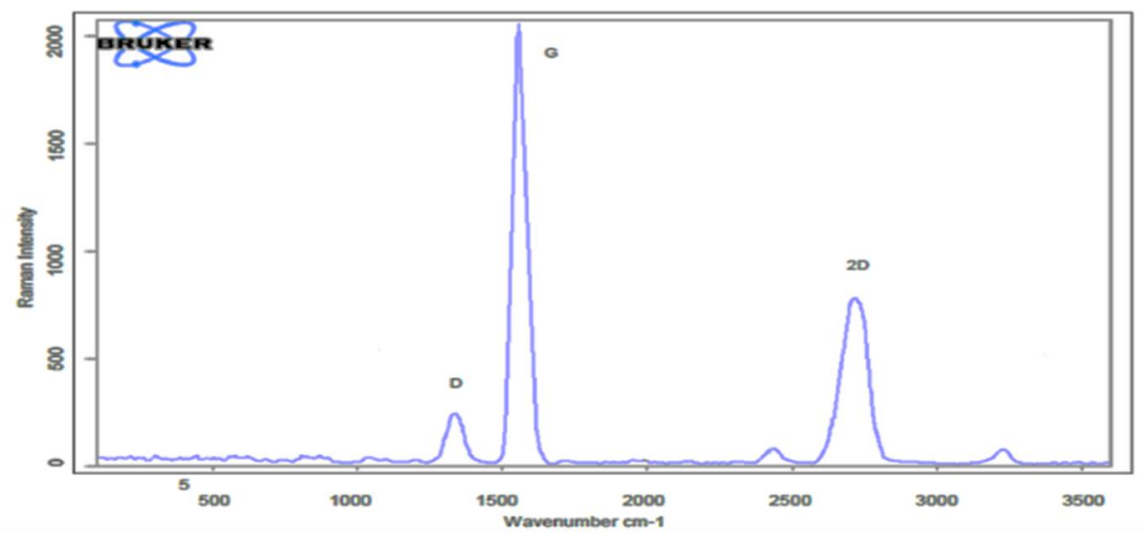

Figure 3: Raman spectra of the prepared graphene 
Figure 6 shows X-ray deflection (XRD): In the case of graphite, a strong XRD peak at $2 \theta=26.6^{\circ}$ and a slight peak at $2 \theta=$ $54.5^{\circ}$ were observed specific to the (002) and (004) planes with d-spacing of $0.34 \mathrm{~nm}$ and $0.19 \mathrm{~nm}$, respectively. And a peak is barely visible at about $2 \theta=4.3^{\circ}$ which corresponds to the refection in the (100) plane of aromatic sheets of graphite. The (002) peak is attributed to the tendency of the aromatic ring carbon reticulated sheets in a three-dimensional configuration, while (100) is attributed to the degree of intensification of the aromatic ring, that is, the size of the carbon mesh slice of the aromatic ring [23].

Figure 7 shows the pattern of graphene depicts a distinctive peak of (002) at $26.2^{\circ}$ with a weaker compared to graphite's peak. The decrease in the intensity of (002) a plane peak indicates large exfoliation of graphite to graphene nanosheets [24].

Fourier transform infrared spectroscopy (FTIR) is a method for obtaining an infrared spectrum of absorption, emission, and photoconductivity of solids, liquids, and gases. It is used to detect various functional groups in the structure of a substance [25].

The (FTIR) patterns of graphite and as-exfoliated graphene are identical, as shown in Figure 8, with only a few absorption signals due to differences in the state of charges between carbon atoms, indicating that no oxygen-containing groups or additional groups were added during the exfoliation process. Consequently, the obtained graphene is relatively free of defects [26].
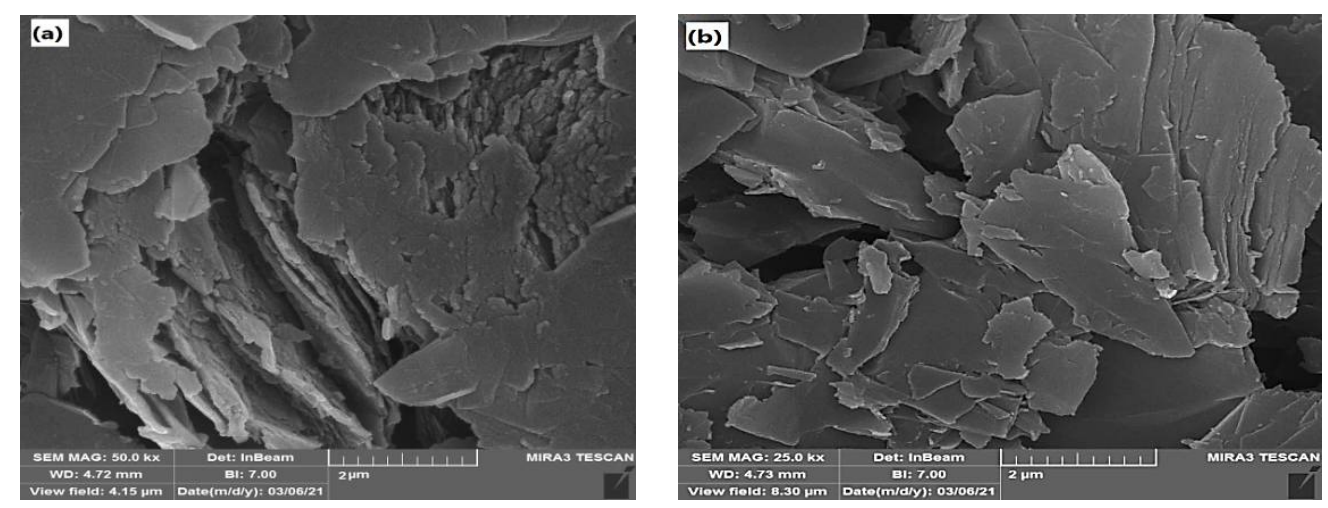

Figure 4: (a) SEM image of Graphite (b) SEM image of graphene
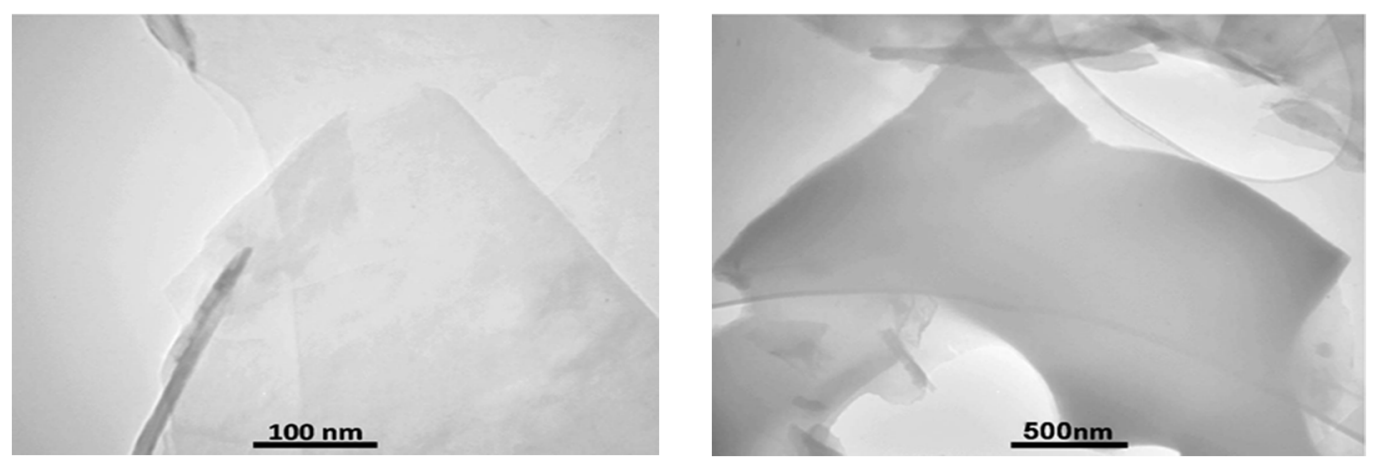

Figure 5: TEM images of the prepared graphene

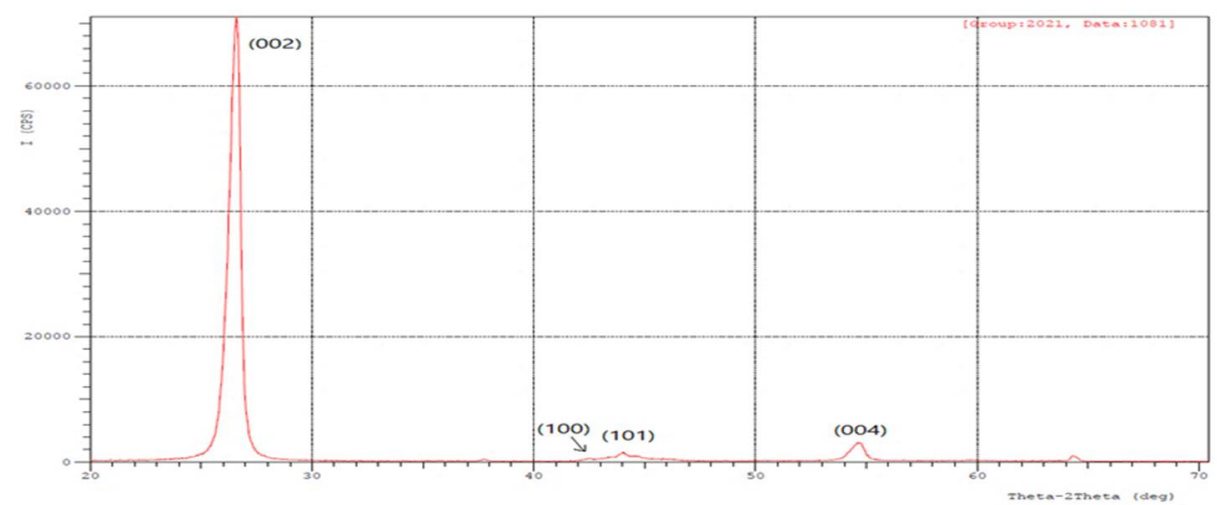

Figure 6: (XRD) pattern of the graphite sample 


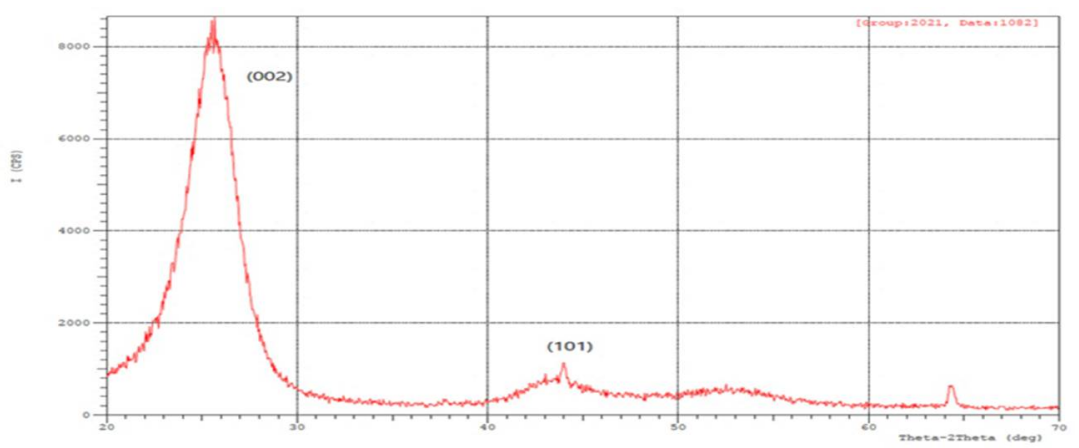

Figure 7: (XRD) pattern of the graphene sample synthesis from graphite flakes

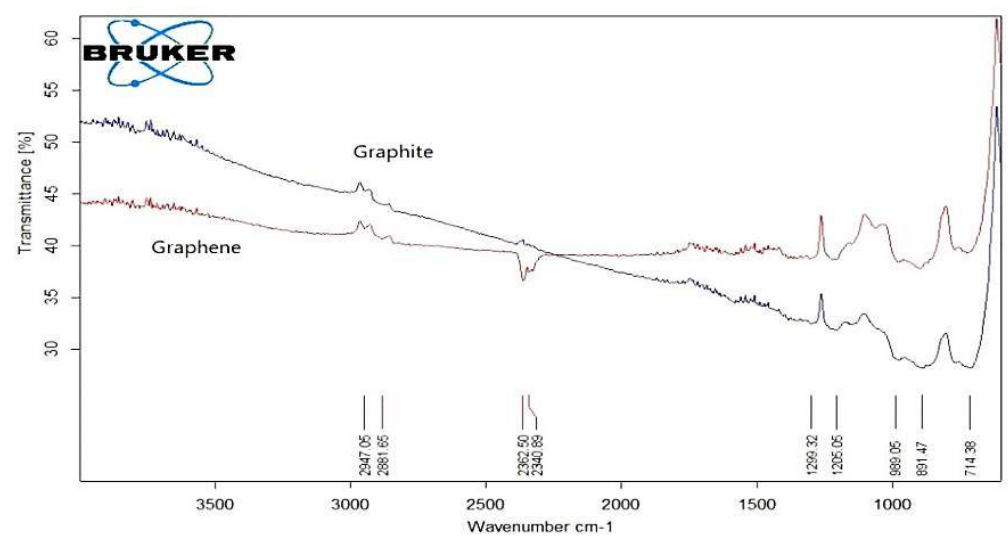

Figure 8: Represents FTIR spectra of graphite and graphene

\section{Conclusions}

Multilayered graphene sheets were synthesized via a series of mechanical exfoliation methods. The dry ball milling process was used. Thereafter, the milling was followed by shear force and then supported by ultra-sonication to reach the lowest level of the graphene sheets.

1) SEM and TEM The images showed the paths involved in the mechanical exfoliation series having enough energy to overcome the Van der Waals forces between the graphene sheets. The thickness of the examined multilayer graphene sheet was estimated at nano-size as well.

2) When compared to graphite, Raman spectra emphasize the production of graphene with the fewest number of layers.

3) Obtained results used both XRD and FTIR characterization, graphene prepared from graphite flakes has the fewest number of layers and relatively few defects.

Overall, the methodology used in this research illustrated a potential approach of the production of low-cost graphene at a high production rate, which is suited for a variety of prospective future applications.

\section{Author contribution}

All authors contributed equally to this work.

Funding

This research received no specific grant from any funding agency in the public, commercial, or not-for-profit sectors.

Data availability statement

The data that support the findings of this study are available on request from the corresponding author.

Conflicts of interest

The authors declare that there is no conflict of interest.

\section{References}

[1] A. M. Rao, M. S. Dresselhaus, Nanostructured forms of carbon: an overview, Nanostructured. Carbon. Adv. Appl., Springer., $1^{\text {st }}$ edition, Netherlands, 24 (2001) 3-24. https://doi.org/10.1007/978-94-010-0858-7_1

[2] T. C. Dinadayalane , J. Leszczynski, Fundamental structural, electronic, and chemical properties of carbon nanostructures: graphene, fullerenes, carbon nanotubes, and their derivatives, Comput. Chem., 2(2012) 793-867. https://doi.org/10.1007/978-94-007-0711-5_22

[3] K. I. Tserpes, N. Silvestre, Modeling of carbon nanotubes, graphene and their composites, Springer, 188(2014).

[4] K. S. Novoselov, A. K. Geim, S. V. Morozov, D Jiang, Y. Zhang, S. V. Dubonos, I. V. Grigorieva, A. A. Firsov, Electric field effect in atomically thin carbon films, Science, 306 (2004) 666-669. https://doi.org/10.1126/science.1102896 
[5] J. Yao, Y. Sun, M. Yang, Y. Duan, Chemistry, physics and biology of graphene-based nanomaterials: new horizons for sensing, imaging and medicine, J. Mater. Chem., 22 (2012) 14313-14329. https://doi.org/10.1039/C2JM31632C

[6] M. I. Katsnelson, Graphene: carbon in two dimensions, Materials today, 10 (2007) 20-27. https://doi.org/10.1016/S13697021(06)71788-6

[7] U. K. Sur, Graphene: a rising star on the horizon of materials science, Int. J. Electrochem., 12 (2012)1-12. https://doi.org/10.1155/2012/237689

[8] S. Ganguly, D. Banerjee, K. Kargupta, Nanotechnology and nanomaterials for new and sustainable energy engineering, Proceedings of the international conference nanomaterials: applications and properties, 1 (2012)1-5.

[9] S. K. Tiwari, V. Kumar, A. Huczko, R. Oraon, A. De Adhikari, G. C. Nayak, Magical allotropes of carbon: prospects and applications, Crit. Rev. Solid .State .Mater. Sci., 41 (2016) 257-317. https://doi.org/10.1080/10408436.2015.1127206

[10] W. Choi, I. Lahiri, R. Seelaboyina, Y. S. Kang, Synthesis of graphene and its applications: a review, Crit. Rev. Solid .State. Mater. Sci., 35 (2010)52-71. https://doi.org/10.1080/10408430903505036

[11] I. Khalil, N. M. Julkapli, W. A. Yehye, W. J. Basirun, S. K. Bhargava, Graphene-gold nanoparticles hybrid-synthesis, functionalization, and application in a electrochemical and surface-enhanced raman scattering biosensor, Mater., 9 (2016) 406. https://doi.org/10.3390/ma9060406

[12] W. Zhao, M. Fang, F. Wu, H. Wu, L. Wang, G. Chen, Preparation of graphene by exfoliation of graphite using wet ball milling, J. Mater. Chem., 20 (2010) 5817-5819. doi:https://doi.org/10.1039/c0jm01354d

[13] E.Varrla, K. R. Paton, C. Backes, A. Harvey, R. J. Smith, J. McCaule , J. N. Coleman, Turbulence-assisted shear exfoliation of graphene using household detergent and a kitchen blender, Nanoscale, 6 (2014) 11810-11819.

https://doi.org/10.1039/C4NR03560G

[14] Z. Ismail, N. F. A. Kassim, A. H. Abdullah, A. S. Z. Abidin, F. S. Ismail, K. Yusoh, Black tea assisted exfoliation using a kitchen mixer allowing one-step production of graphene, Mater. Res. Express., 4 (2017) 1-11. http://dx.doi.org/ $\underline{10.1088 / 2053-1591 / \mathrm{aa} 7 \mathrm{ae} 2}$

[15] Y. Z. N. Htwe, W. S. Chow, Y. Suda, A. A. Thant, M. Mariatti, Effect of electrolytes and sonication times on the formation of graphene using an electrochemical exfoliation process, Appl. Surf. Sci., 469 (2019) 951-961. https://doi.org/10.1016/j.apsusc.2018.11.029

[16] G. Huang, C. Lv, J. He, X. Zhang, C. Zhou, P. Yang, H. Huang, Study on preparation and characterization of graphene based on ball milling method, J. Nanomater., (2020) 1-11. http://dx.doi.org/ 10.1155/2020/2042316

[17] P. Kun, F. Wéber, C. Balázsi, Preparation and examination of multilayer graphene nanosheets by exfoliation of graphite in high efficient attritor mill,Cent. Eur. J. Chem., 9 (2011) 47-51. https://doi.org/10.2478/s11532-010-0137-5

[18] T. Xing, J. Sunarso, W. Yang, Y. Yin, A. M. Glushenkov, L. H. Li , Y. Chen, Ball milling: a green mechanochemical approach for synthesis of nitrogen doped carbon nanoparticles, Nanoscale, 5 (2013) 7970-7976. https://doi.org/10.1039/C3NR02328A

[19] S. Yousef, A. Mohamed, M. Tatariants, Mass production of graphene nanosheets by multi-roll milling technique, Tribol. Int., 121 (2018) 54-63. https://doi.org/10.1016/j.triboint.2018.01.040

[20] M. Yi, Z. Shen, Kitchen blender for producing high-quality few-layer graphene, Carbon, 78 (2014) $622-626$. https://doi.org/10.1016/j.carbon.2014.07.035

[21] A. Ciesielski , P. Samori, Graphene via sonication assisted liquid-phase exfoliation, Chem. Soc. Rev., 43 (2014) 381398. https://doi.org/10.1039/C3CS60217F

[22] I. Childres, L. A. Jauregui, W. Park, H. Cao, Y. P. Chen, Raman spectroscopy of graphene and related materials, New Dev. Phot. Mater. Res., 1 (2013) 1-20.

[23] T. Qiu, J.G. Yang, X.-J. Bai, Y.L. Wang, The preparation of synthetic graphite materials with hierarchical pores from lignite by one-step impregnation and their characterization as dye absorbents, RSC Adv., 9 (2019) 12737-12746. https://doi.org/10.1039/C9RA00343F

[24] M. Matsumoto, Y. Saito, C. Park, T. Fukushima, T. Aida, Ultrahigh-throughput exfoliation of graphite into pristine 'single-layer' graphene using microwaves and molecularly engineered ionic liquids, Nat. Chem., 7 (2015) $730-736$. https://doi.org/10.1038/nchem.2315

[25] D.L. Sparks, A.L. Page, P.A. Helmke, R.H. Loeppert, P. N. Soltanpour, M. A. Tabatabai, C. T. Johnston, M. E. Sumner, Methods Soil Analysis: Part 3 Chemical Methods, Soil Science Society of America, 1st edition, USA, 1, Ch.10(1996)269321. https://doi.org/10.2136/sssabookser5.3

[26] V. Ţucureanu, A. Matei, A. M. Avram, FTIR spectroscopy for carbon family study, Crit. Rev. Anal. Chem., 46 (2016) 502-520. https://doi.org/10.1080/10408347.2016.1157013 